\title{
A Synchrotron Self-Compton Scenario for the Very High Energy $\gamma$-ray Emission of the Intermediate BL Lacertae object W Comae
}

\author{
Jin Zhang ${ }^{1,2}$ \\ 1 National Astronomical Observatories/Yunnan Observatory, Chinese Academy of Sciences, Kunming \\ 650011, China; jinzhang@ynao.ac.cn \\ 2 The Graduate School of Chinese Academy of Sciences
}

\begin{abstract}
W Comae has significant variability in multi-wavelengthes, from the radio to the gamma-ray bands. A bright outburst in the optical and X-ray bands was observed in 1998, and most recently, a strong TeV flare was detected by VERITAS in 2008. It is the first $\mathrm{TeV}$ intermediate-frequency-peaked BL Lacertae (IBL) source. I find that both the broadband spectral energy distributions (SEDs) quasi-simultaneously obtained during the $\mathrm{TeV}$ flare and during the optical/X-ray outburst are well fit by using a single-zone synchrotron + synchrotron-self-Compton (SSC) model. The satisfactory fitting requires a large beaming factor, i.e., $\delta \sim 25$ and $\delta \sim 20$ for the TeV flare and the optical/X-ray outburst, respectively, suggesting that both the optical/X-ray outburst and the TeV flare are from a relativistic jet. The size of the emission region of the $\mathrm{TeV}$ flare is three times larger than that of the optical/Xray outburst, and the strength of the magnetic field for the TeV flare is $\sim 14$ times smaller than that of the X-ray/optical outburst, likely indicating that the region of the $\mathrm{TeV}$ flare is more distant from the core than that of the X-ray/optical outburst. The IC component of the $\mathrm{TeV}$ flare peaks at around $1.3 \mathrm{GeV}$, but it is around $20 \mathrm{MeV}$ for the $\mathrm{X}$-ray/optical outburst, lower than that for the $\mathrm{TeV}$ flare with 2 orders of magnitude. The model predicts that the optical/X-ray outburst might be accompanied by a strong $\mathrm{MeV} / \mathrm{GeV}$ emission, but the $\mathrm{TeV}$ flare may be not associated with the X-ray/optical outburst. The $\mathrm{GeV}$ emission is critical to characterize the SEDs of the optical/X-ray outburst and the TeV flare. The predicted GeV flux is above the sensitivity of Fermi/LAT, and it could be verified with the observations by Fermi/LAT in near future.
\end{abstract}

Key words: BL Lacertae objects: individual: W Comae-Gamma rays : observations-Gamma rays : theory-radiation mechanisms: non-thermal

\section{INTRODUCTION}

The continuum emission of active galactic nuclei (AGNs) is both highly luminous and rapidly variable, especially for a sub-class of blazars. The radiation of blazars is dominated by emission from a relativistic jet oriented close to the line of sight (Blandford \& Rees 1978). Their broad band spectral energy distributions (SEDs) are characterized by two broad, well separated components. It is well believed that the lower one is produced by the synchrotron process, and the higher one could be due to inverse Compton (IC) scattering of the same electron population (e.g., Ulrich et al. 1997; Urry 1999). According to the location of the synchrotron hump, blazars are classified as flat-spectrum radio quasars (FSRQs), low-frequency-peaked BL Lac objects (LBLs) with a synchrotron radiation peak at the IR/optical regime, and high-frequencypeaked BL Lac objects (HBLs) with a synchrotron radiation peak at the X-ray band (Giommi \& Padovani 1994; Ulrich et al. 1997). The intermediate-frequency-peaked BL Lac objects (IBLs) fill in the gap between LBLs and HBLs. 
Blazars show significant variability in multi-frequency, from the radio to the gamma-ray bands, even in the $\mathrm{TeV}$ gamma-ray band. Gamma-ray emission is an important emission component for the SEDs of blazars, since the radiation in this band is comparable to the total radiation power of the sources and even higher than that in the rest of the other energy bands. It plays important roles on understanding the radiation mechanisms and the emission regions of Blazars (e.g., Catanese \& Weekes 1999). Considerable samples of $\mathrm{GeV}-\mathrm{TeV}$ blazars have been obtained with both the spaced-based instruments and ground-based Cherenkov telescopes. For examples, the EGRET instrument on board the Compton Gamma Ray Observatory (CGRO) identified a sample of some MeV/GeV blazars. Most of them are FSRQs and LBLs (Hartman et al. 1999; Mattox et al. 2001). The imaging atmospheric Cherenkov telescopes (IACTs) have established a sample of more than 20 blazars as very high energy (VHE) $\gamma$-ray radiation sources. Almost all of them belong to HBLs. W Comae (W Com, ON 231, 1219+285), an IBL at redshift $z=0.102$, has been long considered as a VHE gamma-ray source (Kerrick et al. 1995). More recently, a TeV flare of this object was detected with VERITAS, an array of four imaging atmospheric-Cherenkov telescopes (Acciari et al. 2008). This is the first $\mathrm{TeV}$ detection from an IBL source. In this paper, I investigate the properties of the VHE emission from this source.

The historic optical light-curve of W Com shows three major outbursts peaking in March 1995, February 1996, and January 1997. The source brightness reached the highest magnitude in April 1998 ( $R=12.2$; Tosti et al. 1999; Massaro et al. 1999), which was ever observed since 194(1]. It was about 3 times brighter than the optical outbursts observed in the previous years and a factor of 60 than the minimum brightness $B=17.5$ in 1972 (Tosti et al. 1999; Massaro et al. 1999). A never detected high polarization was also observed at the same time (Tosti et al. 1999), which indicates a non-thermal origin of the optical outburst. The source was also detected in infrared band by IRAS, the Infrared Astronomical Satellite (Impey \& Neugebauer 1988).

In the X-ray band, W Com was observed by Einstein/IPC in June 1980 (Worrall \& Wilkes 1990) and by ROSAT/PSPC in June 1991 (Lamer et al. 1996; Comastri et al. 1997), with detections of a flux density $\sim 1 \mu \mathrm{Jy}$ and $0.4 \mu \mathrm{Jy}$ at $1 \mathrm{keV}$, respectively. The derived energy spectral index from the ROSAT/PSPC observation is $\alpha \sim 1.2$. The observation with XTE in a multi-wavelength campaign in February 1996 yielded only an upper limit (Maisack et al. 1997). The source was in a high state in the X-ray band during the strongest optical flare in 1998 (Tagliaferri et al. 2000). The observation with the BeppoSAX satellite derived a well-defined two-component feature in the X-ray spectrum, which is explained as a synchrotron component and an IC component (Tagliaferri et al. 2000; Böttcher et al. 2002). Moreover, the source showed a significant variation in the soft X-ray band (0.1-4 keV), but no similar behavior in the hard X-ray band (4-10 keV).

In the gamma-ray band, W Com was first observed with CGRO/EGRET during 1991-1992. The observed gamma-ray spectrum is extremely hard, with $\alpha \sim 0.4 \pm 0.4$ (Montigny et al. 1995; Sreekumar et al. 1996), even harder than that derived in 1995 when the source was in the brightest state in the EGRET band (Tagliaferri et al. 2000). The EGRET observation in February 1996 during a quasi-simultaneous multiwavelength campaign shows that its brightness was weaker than that observed in November 1993 by a factor of 1.5 (Maisack et al. 1997). During April 1998 to May 1998 the source was in a high state in both the optical and X-ray bands, but the gamma-ray emission was only marginally detected by EGRET in March 1998, with a $2.7 \sigma$ significance level (Böttcher et al. 2002). Observations with Whipple/IACT obtained some upper limits in the TeV band in 1993/94 (Kerrick et al. 1995) and 1995/96/99 (Horan et al. 2004). A preliminary $2 \sigma$ upper limit was derived with STACEE in 1998 (Böttcher et al. 2002). Interestingly, a strong TeV flare was detected by VERITAS in the middle of March 2008 (Acciari et al. 2008), but no accompanied ourbursts/flares in the X-ray and optical bands. An X-ray flare was detected about two weeks after the TeV flare (Acciari et al. 2008).

As mentioned above, the outburst/flare events of $\mathrm{W}$ com in the optical/X-ray bands and in the $\mathrm{TeV}$ band seem not to happen simultaneously. It is generally believed that the X-ray/optical emission is produced by the synchrotron radiation. It is a puzzle if the $\mathrm{TeV}$ emission is from the same site as the $\mathrm{X}$-ray/optical emission through an IC scattering by the same electron population of the synchrotron radiation. In this paper, I focus on this issue by fitting the broadband SEDs observed quasi-simultaneously during the $\mathrm{TeV}$ flare in

\footnotetext{
1 Wolf (1916) reported $B=11.5$ in 1901 and 1903.
} 
2008 and during the optical/X-ray outburst phase in 1998 with a single-zone synchrotron+synchrotron- selfCompton (SSC) model (Maraschi et al. 1992; Bloom \& Marscher 1996), and reveal the different properties between the TeV flare and the X-ray/optical outburst by comparing the fitting results.

\section{MODEL}

The broad band SED for blazars is a double-peaked structure. It is well believed that the lower one is produced by the synchrotron process, and the higher one could be due to inverse Compton (IC) scattering of the same electron population (e.g., Ulrich et al. 1997; Urry 1999). The IC scattering photon field could be from the synchrotron radiation themselves, the so-called SSC model (Maraschi et al. 1992; Bloom \& Marscher 1996) or from external radiation fields (EC), such as broad line region (BLR; Sikora et al. 1994; Koratkar et al. 1998), accretion disk (Dermer et al. 1992), torus (Błażejowski et al. 2000), and cosmic microwave background (CMB; Burbidge et al. 1974; Tavecchio et al. 2000). Since those external photon fields are very weak comparing with the synchrotron radiation field for the BL Lac objects, I consider only a synchrotron + SSC model by assuming that the emission region is a homogeneous sphere with dimension $R$ and the electron distribution in energy is a broken power law with indices $p_{1}$ and $p_{2}$ below and above the break energy $\gamma_{b} m_{e} c^{2}$,

$$
N(\gamma)= \begin{cases}N_{0} \gamma^{-p_{1}} & \gamma<\gamma_{b} \\ N_{0} \gamma_{b}^{p_{2}-p_{1}} \gamma^{-p_{2}} & \gamma>\gamma_{b}\end{cases}
$$

where $p_{1,2}=2 \alpha_{1,2}+1, \alpha_{1,2}$ are the spectral indices, and $\gamma$ is the Lorentz factor of the electrons. The frequency of the synchrotron radiation is

$$
\nu_{\text {syn }}=\frac{4}{3} \nu_{B} \gamma^{2} \frac{\delta}{1+z}
$$

where $\nu_{B}=2.8 \times 10^{6} B \mathrm{~Hz}$ is the Larmor frequency in magnetic field $B$ (e.g., Ghisellini et al. 1996) and $\delta$ is the Doppler factor. The synchrotron emissivity $\epsilon_{s}(\nu)$ is calculated with

$$
\epsilon_{s}(\nu)=\frac{1}{4 \pi} \int_{\gamma_{\min }}^{\gamma_{\max }} d \gamma N(\gamma) P_{s}(\gamma, \nu)
$$

Here $P_{s}(\nu, \gamma)$ is the single electron synchrotron emissivity averaged over an isotropic distribution of pitch angles. It is calculated with (Crusius \& Schlickeiser 1986; Ghisellini et al. 1988)

$$
P_{s}(\gamma, \nu)=\frac{3 \sqrt{3}}{\pi} \frac{\sigma_{T} c U_{B}}{\nu_{B}} g^{2}\left\{K_{4 / 3}(g) K_{1 / 3}(g)-\frac{3}{5} g\left[K_{4 / 3}^{2}(g)-K_{1 / 3}^{2}(g)\right]\right\}
$$

where $g=\nu /\left(3 \gamma^{2} \nu_{B}\right), K_{\alpha}$ is the modified $\alpha$-order Bessel function, $\sigma_{T}$ is the Thomson cross-section, and $U_{B}=\frac{B^{2}}{8 \pi}$ is the magnetic field energy density. The synchrotron radiation field $I_{s}(\nu)$ is calculated by the transfer equation,

$$
I_{s}(\nu)=\frac{\epsilon_{s}(\nu)}{k(\nu)}\left[1-e^{-k(\nu) R}\right]
$$

where $k(\nu)$ is the absorption coefficient (Ghisellini \& Svensson 1991).

In the SSC scenario, the relativistic electrons interact with synchrotron radiation photons through the IC scattering. The IC emissivity is calculated by

$$
\epsilon_{c}\left(\nu_{c}\right)=\frac{\sigma_{T}}{4} \int_{\nu_{i}^{\min }}^{\nu_{i}^{\max }} \frac{d \nu_{i}}{\nu_{i}} \int_{\gamma_{1}}^{\gamma_{2}} \frac{d \gamma}{\gamma^{2} \beta^{2}} N(\gamma) f\left(\nu_{i}, \nu_{c}\right) \frac{\nu_{c}}{\nu_{i}} I_{s}\left(\nu_{i}\right)
$$

where $\nu_{i}$ is the frequency of the incident photons emitted by the synchrotron radiation between $\nu_{i}^{\text {min }}$ and $\nu_{i}^{\max }, \beta=v / c, \gamma_{1}$ and $\gamma_{2}$ are the lower and upper limits of the scattering electrons, and $f\left(\nu_{i}, \nu_{c}\right)$ is the spectrum produced by scattering monochromatic photons of frequency $\nu_{i}$ with a single electron (e.g., Rybicki \& Lightman 1979). The medium is transparent for the IC radiation field, so I simply derived $I_{c}\left(\nu_{c}\right)=\epsilon_{c}\left(\nu_{c}\right) R$. 
Table 1 Fitting Parameters

\begin{tabular}{ccccccc}
\hline Epoch & Syn. peak $(\mathrm{Hz})$ & $\alpha_{1}$ & $\alpha_{2}$ & $\delta$ & $\mathrm{B}(\mathrm{G})$ & $\mathrm{R}(\mathrm{cm})$ \\
\hline 2008 & $5.2 \times 10^{14}$ & 0.54 & 1.63 & 24.5 & 0.01 & $6.35 \times 10^{16}$ \\
1998 & $1.0 \times 10^{14}$ & 0.2 & 1.53 & 19.4 & 0.14 & $2.1 \times 10^{16}$ \\
\hline
\end{tabular}

Assuming that $I_{s, c}$ is an isotropic radiation field, the monochromatic luminosity around the source is obtained by

$$
L(\delta \nu)=4 \pi^{2} R^{2} I_{s, c}(\nu) \delta^{3} .
$$

Then the observed flux density is given by

$$
F\left(\nu_{o b s}\right)=\frac{4 \pi^{2} R^{2} I_{s, c}(\nu) \delta^{3}(1+z)}{4 \pi D^{2}},
$$

where $D$ is the luminosity distance of the source and $\nu_{o b s}=\nu \delta /(1+z)$.

In the GeV-TeV regime, the Klein- Nishina effect could be significant. It makes the IC spectrum have a high-energy cut-off. I take this effect into account by using a step function for the energy dependence of the cross section, $\sigma=\sigma_{T}$ for $\gamma x \leq 3 / 4$ and $\sigma=0$ otherwise, where $x=h \nu / m_{e} c^{2}$ (e.g., Tavecchio, et al. 1998; Chiaberge \& Ghisellini 1999). Since W com locates at $z=0.102$, the absorption by the infrared background light and CMB during the gamma-ray photons propagating to Earth is also considered (Stecker et al. 2006).

\section{NUMERICAL RESULTS}

I fit the broadband SEDs observed quasi-simultaneously during the $\mathrm{TeV}$ flare in 2008 and during an optical/X-ray outburst phase in 1998. The SED data of the optical/X-ray flare are taken from Tagliaferri et al. (2000, for the optical and the X-ray data) and Böttcher et al. (2002, for the radio and the the EGRET data). The observed data of the TeV flare in 2008 are from Acciari et al. (2008) and the references therein. The size of emission region is estimated by the variability timescale $t$ with $R=c t \delta$, where $c$ is speed of light. The $t$ value is taken as 10 hours for the $\mathrm{X}$-ray/optical outburst and 1 day for the $\mathrm{TeV}$ flare according to the timescales of the X-ray flare in 1998 and the TeV flare in 2008 (Böttcher et al. 2002; Acciari et al. 2008).

The well-sampled SED data in the radio, optical, X-ray, and TeV bands for the $2008 \mathrm{TeV}$ flare place strong constraints on the physical parameters of the model. Although no $\mathrm{TeV}$ detection was made for the 1998 optical/X-ray outburst, the X-ray spectrum measured by BeppoSAX during the optical/X-ray outburst reveals a clear two-component feature, i.e., a synchrotron component and an IC component (Tagliaferri et al. 2000; Böttcher et al. 2002), which also reliably confines the model parameters. The fitting results are shown in Figure 1, and the model parameters are reported in Table 1. The SED derived by the model is corrected for $\gamma \gamma$ absorption according to the baseline infrared background light case of Stecker et al. (2006). It is found that the model well fits the SEDs. Large beaming factors, $\delta \sim 25$ and $\delta \sim 20$ are required to fit the SEDs, suggesting that both the optical/X-ray outburst and the TeV flare are from a relativistic jet. It is interesting that size of the emission region of the $\mathrm{TeV}$ flare is three times larger than that of the optical/Xray outburst, and the strength of the magnetic field for the TeV flare is $\sim 14$ times smaller than that of the $\mathrm{X}$-ray/optical outburst, likely indicating that the region of the $\mathrm{TeV}$ flare is further away from the core than that of the X-ray/optical outburst. The IC component of the TeV flare peaks at around $10^{24} \mathrm{~Hz}$, but it is around $10^{22} \mathrm{~Hz}$ for the $\mathrm{X}$-ray/optical outburst, lower than that for the TeV flare with 2 orders of magnitude.

\section{CONCLUSION AND DISCUSSION}

The broadband SEDs observed quasi-simultaneously during the TeV flare in 2008 and during the optical/Xray outburst phase in 1998 are fitted with the single-zone synchrotron+SSC model. In the model, the IC radiation of the external photon fields (EC) is not taken into account since the CMB/BLR/torus/accrection 


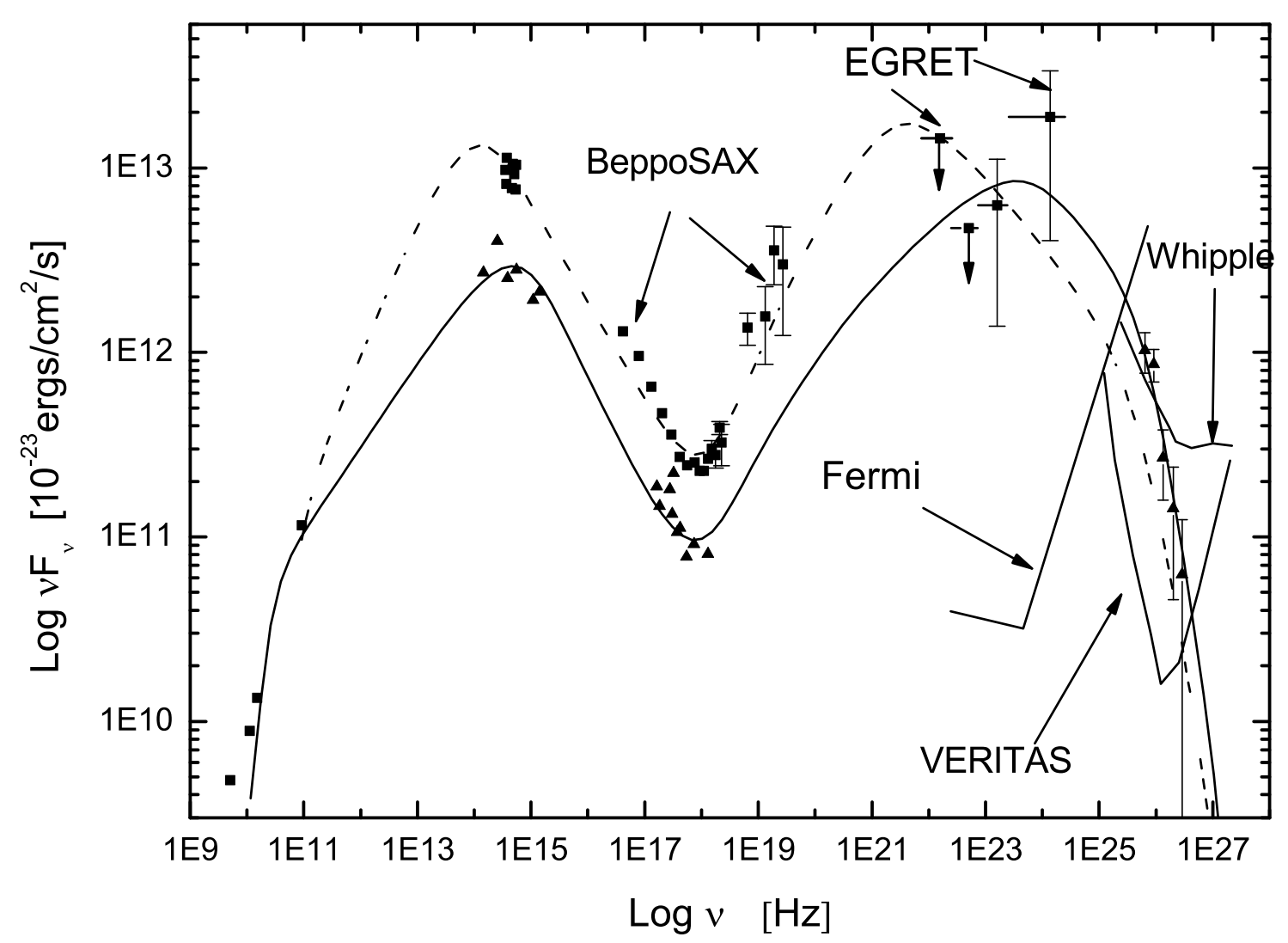

Fig. 1 Broadband SEDs of the optical/X-ray outburst in 1998 (squares) and of the TeV flare in 2008 (triangles) with the model fits. The SED data of the optical/X-ray outburst are taken from Tagliaferri et al. (2000, for the optical and the X-ray data) and Böttcher et al. (2002, for the radio and the the EGRET data). The observed data of the TeV flare in 2008 are from Acciari et al. (2008) and the references therein. The model parameters are $\delta=24.5, B=0.01 \mathrm{G}$, and $R=$ $6.35 \times 10^{16} \mathrm{~cm}$ for the fit to the SED of the $2008 \mathrm{TeV}$ flare (solid line), and $\delta=19.4, B=0.14$ $\mathrm{G}$, and $R=2.1 \times 10^{16} \mathrm{~cm}$ for the fit to the SED of the 1998 optical/X-ray outburst (dashed line). The sensitivity curves for Whipple, VERITAS, and Fermi are also shown as marked in the figure.

disk photon fields are weak comparing with the synchrotron radiation field for a BL Lac object. The results show that the SEDs can be described by the synchrotron + SSC leptonic jet model.

The satisfactory fitting requires a large beaming factor, i.e., $\delta \sim 25$ and $\delta \sim 20$ for the TeV flare and the optical/X-ray outburst, respectively, suggesting that both the optical/X-ray outburst and the TeV flare are from a relativistic jet. The strength of the magnetic field for the $\mathrm{TeV}$ flare is $\sim 14$ times smaller than that of the X-ray/optical outburst, likely indicating that the emission region is more distant from the core in 2008 than in 1998, since the magnetic field $B$ decreases along the jet. Electrons can be reaccelerated after leaving the inner jet, hence the peak frequencies of synchrotron radiation in the kiloparsec-scale jets could be more than 100 times larger than those in the inner jets (e.g., Bai \& Lee 2003). The results favor this idea.

Almost the entire flare in 2008, which lasts four nights, was recorded by VERITAS. The simultaneous observations in the X-ray band did not detect any outburst/flare events. An X-ray flare following the TeV 
flare after two weeks was observed, with a peak flux 4 times higher than that observed during the TeV flare. This fact shows that the TeV flare and the X-ray flare may be not simultaneous. As shown in Figure 1, the IC component peaks around $10^{22} \mathrm{~Hz}$ for the SED during an optical/X-ray outburst, which is 2 order of magnitudes lower than that for the SED during the TeV flare. The GeV observation thus is critical to characterize the SEDs of both the optical/X-ray outburst and the TeV flare. The fitting results suggest that the optical/X-ray outburst may accompany a strong $\mathrm{MeV} / \mathrm{GeV}$ flare. The predicted flux at $\mathrm{GeV}$ is much above the sensitivity of Fermi/LAT, and W com is a selected target for Fermi/LAT. The model could be verified with the Fermi/LAT observation.

In the $\mathrm{TeV}$ band, the predicted $\mathrm{TeV}$ flux by the model for the optical/X-ray outburst in 1998 is lower than the sensitivity of Whipple/IACT. This may be the reason why no detection was obtained with Whipple/IACT in 1998. Although the predicted TeV flux is marginally over the sensitivity of VERITAS, it is much lower than the observed $\mathrm{TeV}$ flux in 2008 . .

Acknowledgements I appreciate the referee for his valuable suggestions. I thank Jinming Bai, Liang Chen and Hongtao Liu for their helpful discussions. This work is supported by the National Natural Science Foundation of China (Grants 10573030 and 10533050).

\section{References}

Acciari, V. A. et al., 2008, ApJ, 684, L73

Bai, J. M., Lee, M. G., 2003, ApJ, 585, L113

Böttcher, M. et al., 2002, ApJ, 581, 143

Blandford, R. D., Rees, M. J., 1978, In: Pittsbur gh Conference on BL Lac Objects, Pittsburgh, University of Pittsburgh, 1978, p. 328

Bloom, S. D., Marscher, A. P., 1996, ApJ, 461, 657

BŁażejowski, M. et al., 2000, ApJ, 545, 107

Burbidge, G. R. et al., 1974, ApJ, 193, 43

Catanese, M., Weekes, R. M., 1999, PASP, 111, 1193

Chiaberge, M., Ghisellini, G., 1999, MNRAS, 306, 551

Comastri, A. et al., 1997, ApJ, 480, 534

Crusius, A., Schlickeiser, R., 1986, A\&A, 164, L16

Dermer, C. D. et al., 1992, A\&A, 256, L27

Ghisellini, G. et al., 1988, ApJ, 334, L5

Ghisellini, G., Svensson, R., 1991, MNRAS, 252, 313

Ghisellini, G. et al., 1996, A\&AS, 120, 503

Giommi, P., Padovani, P., 1994, MNRAS, 268, L51

Hartman, R. C. et al., 1999, ApJS, 123, 79

Horan, D. et al., 2004, ApJ, 603, 51

Impey, C. D., Neugebauer, G., 1988, AJ, 95, 307

Kerrick, A. D. et al., 1995, ApJ, 452, 588

Koratkar, A. P. et al., 1998, ApJ, 492, 173

Lamer, G., Brunner, H., Staubert, R., 1996, A\&A, 311, 384

Maisack, M. et al., 1997, preprint, astro-ph/9706243

Maraschi, L. et al., 1992, ApJ, 397, L5

Massaro, E. et al., 1999, A\&A, 342, L49

Mattox, J. R. et al., 2001, ApJS, 135, 155

von Montigny, C. et al., 1995, ApJ, 440, 525

Rybicki, G., Lightman, A. P., 1979, Radiative Process in Astrophysics. Wiley Interscience, New York, P. 204

Sikora, M. et al., 1994, ApJ, 421, 153 
Sreekumar, P. et al., 1996, ApJ, 464, 628

Stecker, F. W. et al., 2006, ApJ, 648, 774

Tagliaferri, G. et al., 2000, A\&A, 354, 431

Tavecchio, F., et al., 1998, ApJ, 509, 608

Tavecchio, F., et al., 2000, ApJ, 544, L23

Tosti, G. et al., 1999, ASPS, 159, 149

Ulrich, M. H. et al., 1997, ARA\&A, 35, 445

Urry, C. M., 1999, Astropart. Phys., 11, 159

Wolf, M., 1916, Astron Nachr., 202, 415

Worrall, D. M., Wilkes, B. J., 1990, ApJ, 360, 396

This was prepared with the RAA LATEX macro v1.0. 
Research in Astronomy and Astrophysics manuscript no.

(LTEX: 67.tex.tex; printed on November 20, 2018; 6:25)

\title{
A Synchrotron Self-Compton Scenario for the Very High Energy $\gamma$-ray Emission of the Intermediate BL Lacertae object W Comae
}

\author{
Jin Zhang ${ }^{1,2}$ \\ ${ }^{1}$ National Astronomical Observatories/Yunnan Observatory, Chinese Academy of Sciences, Kunming \\ 650011, China; jinzhang@ynao.ac.cn \\ 2 The Graduate School of Chinese Academy of Sciences
}

\begin{abstract}
W Comae has significant variability in multi-wavelengthes, from the radio to the gamma-ray bands. A bright outburst in the optical and X-ray bands was observed in 1998, and most recently, a strong TeV flare was detected by VERITAS in 2008. It is the first $\mathrm{TeV}$ intermediate-frequency-peaked BL Lacertae (IBL) source. I find that both the broadband spectral energy distributions (SEDs) quasi-simultaneously obtained during the $\mathrm{TeV}$ flare and during the optical/X-ray outburst are well fit by using a single-zone synchrotron + synchrotron-self-Compton (SSC) model. The satisfactory fitting requires a large beaming factor, i.e., $\delta \sim 25$ and $\delta \sim 20$ for the TeV flare and the optical/X-ray outburst, respectively, suggesting that both the optical/X-ray outburst and the $\mathrm{TeV}$ flare are from a relativistic jet. The size of the emission region of the $\mathrm{TeV}$ flare is three times larger than that of the optical/Xray outburst, and the strength of the magnetic field for the $\mathrm{TeV}$ flare is $\sim 14$ times smaller than that of the X-ray/optical outburst, likely indicating that the region of the $\mathrm{TeV}$ flare is more distant from the core than that of the X-ray/optical outburst. The IC component of the $\mathrm{TeV}$ flare peaks at around $1.3 \mathrm{GeV}$, but it is around $20 \mathrm{MeV}$ for the X-ray/optical outburst, lower than that for the $\mathrm{TeV}$ flare with 2 orders of magnitude. The model predicts that the optical/X-ray outburst might be accompanied by a strong $\mathrm{MeV} / \mathrm{GeV}$ emission, but the $\mathrm{TeV}$ flare may be not associated with the X-ray/optical outburst. The $\mathrm{GeV}$ emission is critical to characterize the SEDs of the optical/X-ray outburst and the TeV flare. The predicted $\mathrm{GeV}$ flux is above the sensitivity of Fermi/LAT, and it could be verified with the observations by Fermi/LAT in near future.
\end{abstract}

Key words: BL Lacertae objects: individual: W Comae-Gamma rays : observations-Gamma rays : theory-radiation mechanisms: non-thermal

\section{INTRODUCTION}

The continuum emission of active galactic nuclei (AGNs) is both highly luminous and rapidly variable, 
oriented close to the line of sight (Blandford \& Rees 1978). Their broad band spectral energy distributions (SEDs) are characterized by two broad, well separated components. It is well believed that the lower one is produced by the synchrotron process, and the higher one could be due to inverse Compton (IC) scattering of the same electron population (e.g., Ulrich et al. 1997; Urry 1999). According to the location of the synchrotron hump, blazars are classified as flat-spectrum radio quasars (FSRQs), low-frequency-peaked BL Lac objects (LBLs) with a synchrotron radiation peak at the IR/optical regime, and high-frequencypeaked BL Lac objects (HBLs) with a synchrotron radiation peak at the X-ray band (Giommi \& Padovani 1994; Ulrich et al. 1997). The intermediate-frequency-peaked BL Lac objects (IBLs) fill in the gap between LBLs and HBLs.

Blazars show significant variability in multi-frequency, from the radio to the gamma-ray bands, even in the $\mathrm{TeV}$ gamma-ray band. Gamma-ray emission is an important emission component for the SEDs of blazars, since the radiation in this band is comparable to the total radiation power of the sources and even higher than that in the rest of the other energy bands. It plays important roles on understanding the radiation mechanisms and the emission regions of Blazars (e.g., Catanese \& Weekes 1999). Considerable samples of $\mathrm{GeV}-\mathrm{TeV}$ blazars have been obtained with both the spaced-based instruments and ground-based Cherenkov telescopes. For examples, the EGRET instrument on board the Compton Gamma Ray Observatory (CGRO) identified a sample of some MeV/GeV blazars. Most of them are FSRQs and LBLs (Hartman et al. 1999; Mattox et al. 2001). The imaging atmospheric Cherenkov telescopes (IACTs) have established a sample of more than 20 blazars as very high energy (VHE) $\gamma$-ray radiation sources. Almost all of them belong to HBLs. W Comae (W Com, ON 231, 1219+285), an IBL at redshift $z=0.102$, has been long considered as a VHE gamma-ray source (Kerrick et al. 1995). More recently, a TeV flare of this object was detected with VERITAS, an array of four imaging atmospheric-Cherenkov telescopes (Acciari et al. 2008). This is the first $\mathrm{TeV}$ detection from an IBL source. In this paper, I investigate the properties of the VHE emission from this source.

The historic optical light-curve of W Com shows three major outbursts peaking in March 1995, February 1996, and January 1997. The source brightness reached the highest magnitude in April 1998 ( $R=12.2$; Tosti et al. 1999; Massaro et al. 1999), which was ever observed since 19401. It was about 3 times brighter than the optical outbursts observed in the previous years and a factor of 60 than the minimum brightness $B=17.5$ in 1972 (Tosti et al. 1999; Massaro et al. 1999). A never detected high polarization was also observed at the same time (Tosti et al. 1999), which indicates a non-thermal origin of the optical outburst. The source was also detected in infrared band by IRAS, the Infrared Astronomical Satellite (Impey \& Neugebauer 1988).

In the X-ray band, W Com was observed by Einstein/IPC in June 1980 (Worrall \& Wilkes 1990) and by ROSAT/PSPC in June 1991 (Lamer et al. 1996; Comastri et al. 1997), with detections of a flux density $\sim 1 \mu \mathrm{Jy}$ and $0.4 \mu \mathrm{Jy}$ at $1 \mathrm{keV}$, respectively. The derived energy spectral index from the ROSAT/PSPC observation is $\alpha \sim 1.2$. The observation with XTE in a multi-wavelength campaign in February 1996 yielded only an upper limit (Maisack et al. 1997). The source was in a high state in the X-ray band during the strongest optical flare in 1998 (Tagliaferri et al. 2000). The observation with the BeppoSAX satellite derived 
a well-defined two-component feature in the X-ray spectrum, which is explained as a synchrotron component and an IC component (Tagliaferri et al. 2000; Böttcher et al. 2002). Moreover, the source showed a significant variation in the soft X-ray band (0.1-4 keV), but no similar behavior in the hard X-ray band (4-10 $\mathrm{keV})$.

In the gamma-ray band, W Com was first observed with CGRO/EGRET during 1991-1992. The observed gamma-ray spectrum is extremely hard, with $\alpha \sim 0.4 \pm 0.4$ (Montigny et al. 1995; Sreekumar et al. 1996), even harder than that derived in 1995 when the source was in the brightest state in the EGRET band (Tagliaferri et al. 2000). The EGRET observation in February 1996 during a quasi-simultaneous multiwavelength campaign shows that its brightness was weaker than that observed in November 1993 by a factor of 1.5 (Maisack et al. 1997). During April 1998 to May 1998 the source was in a high state in both the optical and X-ray bands, but the gamma-ray emission was only marginally detected by EGRET in March 1998, with a $2.7 \sigma$ significance level (Böttcher et al. 2002). Observations with Whipple/IACT obtained some upper limits in the TeV band in 1993/94 (Kerrick et al. 1995) and 1995/96/99 (Horan et al. 2004). A preliminary $2 \sigma$ upper limit was derived with STACEE in 1998 (Böttcher et al. 2002). Interestingly, a strong TeV flare was detected by VERITAS in the middle of March 2008 (Acciari et al. 2008), but no accompanied ourbursts/flares in the X-ray and optical bands. An X-ray flare was detected about two weeks after the TeV flare (Acciari et al. 2008).

As mentioned above, the outburst/flare events of $\mathrm{W}$ com in the optical/X-ray bands and in the $\mathrm{TeV}$ band seem not to happen simultaneously. It is generally believed that the X-ray/optical emission is produced by the synchrotron radiation. It is a puzzle if the $\mathrm{TeV}$ emission is from the same site as the $\mathrm{X}$-ray/optical emission through an IC scattering by the same electron population of the synchrotron radiation. In this paper, I focus on this issue by fitting the broadband SEDs observed quasi-simultaneously during the $\mathrm{TeV}$ flare in 2008 and during the optical/X-ray outburst phase in 1998 with a single-zone synchrotron+synchrotron- selfCompton (SSC) model (Maraschi et al. 1992; Bloom \& Marscher 1996), and reveal the different properties between the $\mathrm{TeV}$ flare and the $\mathrm{X}$-ray/optical outburst by comparing the fitting results.

\section{MODEL}

The broad band SED for blazars is a double-peaked structure. It is well believed that the lower one is produced by the synchrotron process, and the higher one could be due to inverse Compton (IC) scattering of the same electron population (e.g., Ulrich et al. 1997; Urry 1999). The IC scattering photon field could be from the synchrotron radiation themselves, the so-called SSC model (Maraschi et al. 1992; Bloom \& Marscher 1996) or from external radiation fields (EC), such as broad line region (BLR; Sikora et al. 1994; Koratkar et al. 1998), accretion disk (Dermer et al. 1992), torus (BŁażejowski et al. 2000), and cosmic microwave background (CMB; Burbidge et al. 1974; Tavecchio et al. 2000). Since those external photon fields are very weak comparing with the synchrotron radiation field for the BL Lac objects, I consider only a synchrotron + SSC model by assuming that the emission region is a homogeneous sphere with dimension $R$ and the electron distribution in energy is a broken power law with indices $p_{1}$ and $p_{2}$ below and above the break energy $\gamma_{b} m_{e} c^{2}$,

$$
N(\gamma)= \begin{cases}N_{0} \gamma^{-p_{1}} & \gamma<\gamma_{b},\end{cases}
$$


where $p_{1,2}=2 \alpha_{1,2}+1, \alpha_{1,2}$ are the spectral indices, and $\gamma$ is the Lorentz factor of the electrons. The frequency of the synchrotron radiation is

$$
\nu_{s y n}=\frac{4}{3} \nu_{B} \gamma^{2} \frac{\delta}{1+z}
$$

where $\nu_{B}=2.8 \times 10^{6} B \mathrm{~Hz}$ is the Larmor frequency in magnetic field $B$ (e.g., Ghisellini et al. 1996) and $\delta$ is the Doppler factor. The synchrotron emissivity $\epsilon_{s}(\nu)$ is calculated with

$$
\epsilon_{s}(\nu)=\frac{1}{4 \pi} \int_{\gamma_{\min }}^{\gamma_{\max }} d \gamma N(\gamma) P_{s}(\gamma, \nu)
$$

Here $P_{s}(\nu, \gamma)$ is the single electron synchrotron emissivity averaged over an isotropic distribution of pitch angles. It is calculated with (Crusius \& Schlickeiser 1986; Ghisellini et al. 1988)

$$
P_{s}(\gamma, \nu)=\frac{3 \sqrt{3}}{\pi} \frac{\sigma_{T} c U_{B}}{\nu_{B}} g^{2}\left\{K_{4 / 3}(g) K_{1 / 3}(g)-\frac{3}{5} g\left[K_{4 / 3}^{2}(g)-K_{1 / 3}^{2}(g)\right]\right\}
$$

where $g=\nu /\left(3 \gamma^{2} \nu_{B}\right), K_{\alpha}$ is the modified $\alpha$-order Bessel function, $\sigma_{T}$ is the Thomson cross-section, and $U_{B}=\frac{B^{2}}{8 \pi}$ is the magnetic field energy density. The synchrotron radiation field $I_{s}(\nu)$ is calculated by the transfer equation,

$$
I_{s}(\nu)=\frac{\epsilon_{s}(\nu)}{k(\nu)}\left[1-e^{-k(\nu) R}\right],
$$

where $k(\nu)$ is the absorption coefficient (Ghisellini \& Svensson 1991).

In the SSC scenario, the relativistic electrons interact with synchrotron radiation photons through the IC scattering. The IC emissivity is calculated by

$$
\epsilon_{c}\left(\nu_{c}\right)=\frac{\sigma_{T}}{4} \int_{\nu_{i}^{\min }}^{\nu_{i}^{\max }} \frac{d \nu_{i}}{\nu_{i}} \int_{\gamma_{1}}^{\gamma_{2}} \frac{d \gamma}{\gamma^{2} \beta^{2}} N(\gamma) f\left(\nu_{i}, \nu_{c}\right) \frac{\nu_{c}}{\nu_{i}} I_{s}\left(\nu_{i}\right),
$$

where $\nu_{i}$ is the frequency of the incident photons emitted by the synchrotron radiation between $\nu_{i}^{\min }$ and $\nu_{i}^{\max }, \beta=v / c, \gamma_{1}$ and $\gamma_{2}$ are the lower and upper limits of the scattering electrons, and $f\left(\nu_{i}, \nu_{c}\right)$ is the spectrum produced by scattering monochromatic photons of frequency $\nu_{i}$ with a single electron (e.g., Rybicki \& Lightman 1979). The medium is transparent for the IC radiation field, so I simply derived $I_{c}\left(\nu_{c}\right)=\epsilon_{c}\left(\nu_{c}\right) R$.

Assuming that $I_{s, c}$ is an isotropic radiation field, the monochromatic luminosity around the source is obtained by

$$
L(\delta \nu)=4 \pi^{2} R^{2} I_{s, c}(\nu) \delta^{3} .
$$

Then the observed flux density is given by

$$
F\left(\nu_{o b s}\right)=\frac{4 \pi^{2} R^{2} I_{s, c}(\nu) \delta^{3}(1+z)}{4 \pi D^{2}},
$$

where $D$ is the luminosity distance of the source and $\nu_{o b s}=\nu \delta /(1+z)$.

In the GeV-TeV regime, the Klein- Nishina effect could be significant. It makes the IC spectrum have a high-energy cut-off. I take this effect into account by using a step function for the energy dependence of the cross section, $\sigma=\sigma_{T}$ for $\gamma x \leq 3 / 4$ and $\sigma=0$ otherwise, where $x=h \nu / m_{e} c^{2}$ (e.g., Tavecchio, et al. 1998; Chiaberge \& Ghisellini 1999). Since W com locates at $z=0.102$, the absorption by the infrared background light and CMB during the gamma-ray photons propagating to Earth is also considered (Stecker 
Table 1 Fitting Parameters

\begin{tabular}{ccccccc}
\hline Epoch & Syn. peak $(\mathrm{Hz})$ & $\alpha_{1}$ & $\alpha_{2}$ & $\delta$ & $\mathrm{B}(\mathrm{G})$ & $\mathrm{R}(\mathrm{cm})$ \\
\hline 2008 & $5.2 \times 10^{14}$ & 0.54 & 1.63 & 24.5 & 0.01 & $6.35 \times 10^{16}$ \\
1998 & $1.0 \times 10^{14}$ & 0.2 & 1.53 & 19.4 & 0.14 & $2.1 \times 10^{16}$ \\
\hline
\end{tabular}

\section{NUMERICAL RESULTS}

I fit the broadband SEDs observed quasi-simultaneously during the $\mathrm{TeV}$ flare in 2008 and during an optical/X-ray outburst phase in 1998. The SED data of the optical/X-ray flare are taken from Tagliaferri et al. (2000, for the optical and the X-ray data) and Böttcher et al. (2002, for the radio and the the EGRET data). The observed data of the TeV flare in 2008 are from Acciari et al. (2008) and the references therein. The size of emission region is estimated by the variability timescale $t$ with $R=c t \delta$, where $c$ is speed of light. The $t$ value is taken as 10 hours for the $\mathrm{X}$-ray/optical outburst and 1 day for the $\mathrm{TeV}$ flare according to the timescales of the X-ray flare in 1998 and the TeV flare in 2008 (Böttcher et al. 2002; Acciari et al. 2008).

The well-sampled SED data in the radio, optical, X-ray, and TeV bands for the $2008 \mathrm{TeV}$ flare place strong constraints on the physical parameters of the model. Although no TeV detection was made for the 1998 optical/X-ray outburst, the X-ray spectrum measured by BeppoSAX during the optical/X-ray outburst reveals a clear two-component feature, i.e., a synchrotron component and an IC component (Tagliaferri et al. 2000; Böttcher et al. 2002), which also reliably confines the model parameters. The fitting results are shown in Figure 1, and the model parameters are reported in Table 1. The SED derived by the model is corrected for $\gamma \gamma$ absorption according to the baseline infrared background light case of Stecker et al. (2006). It is found that the model well fits the SEDs. Large beaming factors, $\delta \sim 25$ and $\delta \sim 20$ are required to fit the SEDs, suggesting that both the optical/X-ray outburst and the TeV flare are from a relativistic jet. It is interesting that size of the emission region of the $\mathrm{TeV}$ flare is three times larger than that of the optical/Xray outburst, and the strength of the magnetic field for the $\mathrm{TeV}$ flare is $\sim 14$ times smaller than that of the $\mathrm{X}$-ray/optical outburst, likely indicating that the region of the $\mathrm{TeV}$ flare is further away from the core than that of the X-ray/optical outburst. The IC component of the TeV flare peaks at around $10^{24} \mathrm{~Hz}$, but it is around $10^{22} \mathrm{~Hz}$ for the $\mathrm{X}$-ray/optical outburst, lower than that for the TeV flare with 2 orders of magnitude.

\section{CONCLUSION AND DISCUSSION}

The broadband SEDs observed quasi-simultaneously during the TeV flare in 2008 and during the optical/Xray outburst phase in 1998 are fitted with the single-zone synchrotron+SSC model. In the model, the IC radiation of the external photon fields (EC) is not taken into account since the CMB/BLR/torus/accrection disk photon fields are weak comparing with the synchrotron radiation field for a BL Lac object. The results show that the SEDs can be described by the synchrotron + SSC leptonic jet model.

The satisfactory fitting requires a large beaming factor, i.e., $\delta \sim 25$ and $\delta \sim 20$ for the $\mathrm{TeV}$ flare and the optical/X-ray outburst, respectively, suggesting that both the optical/X-ray outburst and the $\mathrm{TeV}$ flare are 


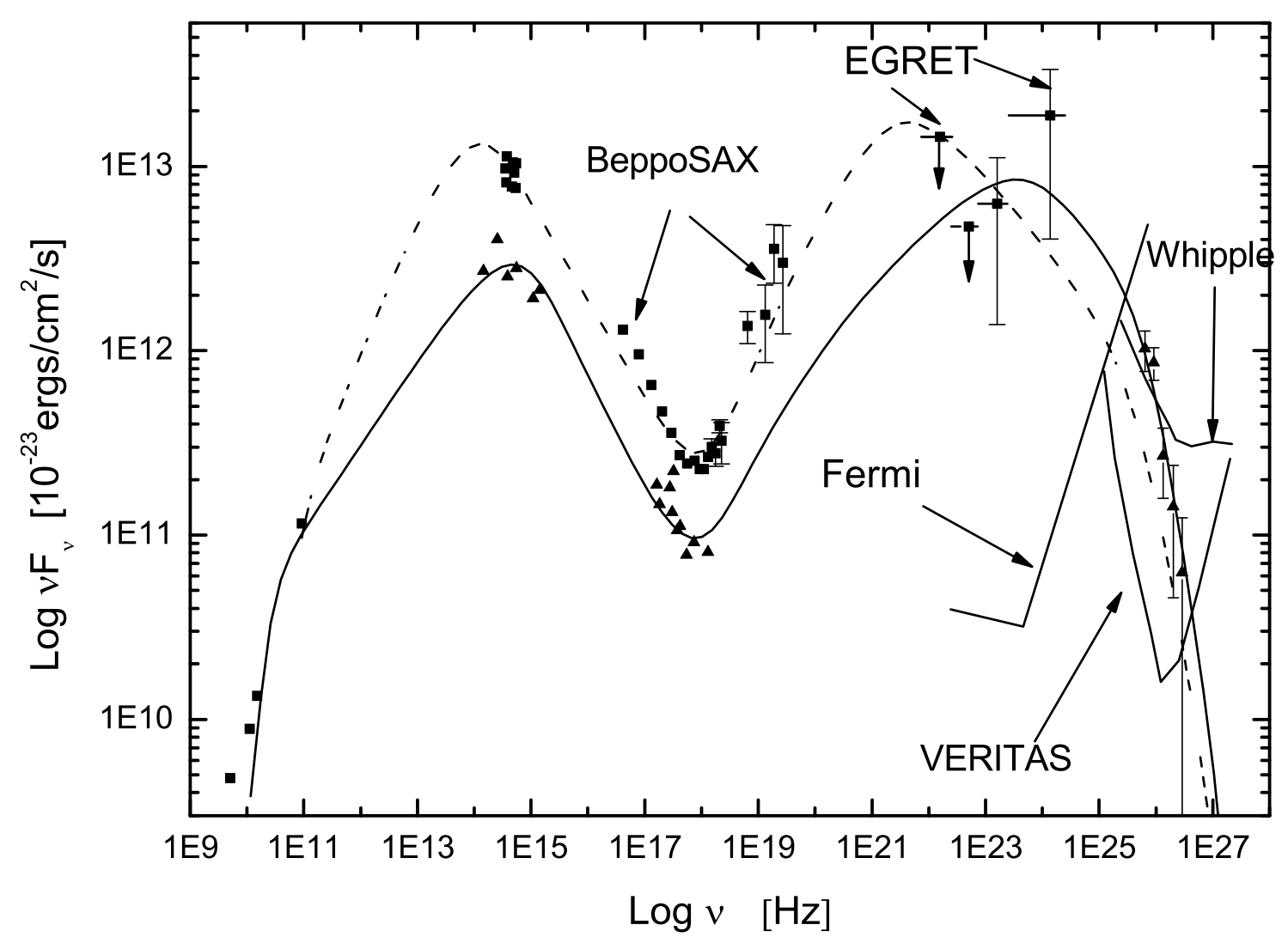

Fig. 1 Broadband SEDs of the optical/X-ray outburst in 1998 (squares) and of the TeV flare in 2008 (triangles) with the model fits. The SED data of the optical/X-ray outburst are taken from Tagliaferri et al. (2000, for the optical and the X-ray data) and Böttcher et al. (2002, for the radio and the the EGRET data). The observed data of the TeV flare in 2008 are from Acciari et al. (2008) and the references therein. The model parameters are $\delta=24.5, B=0.01 \mathrm{G}$, and $R=$ $6.35 \times 10^{16} \mathrm{~cm}$ for the fit to the SED of the $2008 \mathrm{TeV}$ flare (solid line), and $\delta=19.4, B=0.14$ $\mathrm{G}$, and $R=2.1 \times 10^{16} \mathrm{~cm}$ for the fit to the SED of the 1998 optical/X-ray outburst (dashed line). The sensitivity curves for Whipple, VERITAS, and Fermi are also shown as marked in the figure.

the X-ray/optical outburst, likely indicating that the emission region is more distant from the core in 2008 than in 1998, since the magnetic field $B$ decreases along the jet. Electrons can be reaccelerated after leaving the inner jet, hence the peak frequencies of synchrotron radiation in the kiloparsec-scale jets could be more than 100 times larger than those in the inner jets (e.g., Bai \& Lee 2003). The results favor this idea.

Almost the entire flare in 2008, which lasts four nights, was recorded by VERITAS. The simultaneous observations in the X-ray band did not detect any outburst/flare events. An X-ray flare following the $\mathrm{TeV}$ flare after two weeks was observed, with a peak flux 4 times higher than that observed during the TeV flare. This fact shows that the TeV flare and the X-ray flare may be not simultaneous. As shown in Figure 1, the IC component peaks around $10^{22} \mathrm{~Hz}$ for the SED during an optical/X-ray outburst, which is 2 order 
characterize the SEDs of both the optical/X-ray outburst and the $\mathrm{TeV}$ flare. The fitting results suggest that the optical/X-ray outburst may accompany a strong $\mathrm{MeV} / \mathrm{GeV}$ flare. The predicted flux at $\mathrm{GeV}$ is much above the sensitivity of Fermi/LAT, and W com is a selected target for Fermi/LAT. The model could be verified with the Fermi/LAT observation.

In the $\mathrm{TeV}$ band, the predicted $\mathrm{TeV}$ flux by the model for the optical/X-ray outburst in 1998 is lower than the sensitivity of Whipple/IACT. This may be the reason why no detection was obtained with Whipple/IACT in 1998. Although the predicted TeV flux is marginally over the sensitivity of VERITAS, it is much lower than the observed $\mathrm{TeV}$ flux in 2008 . .

Acknowledgements I appreciate the referee for his valuable suggestions. I thank Jinming Bai, Liang Chen and Hongtao Liu for their helpful discussions. This work is supported by the National Natural Science Foundation of China (Grants 10573030 and 10533050).

\section{References}

Acciari, V. A. et al., 2008, ApJ, 684, L73

Bai, J. M., Lee, M. G., 2003, ApJ, 585, L113

Böttcher, M. et al., 2002, ApJ, 581, 143

Blandford, R. D., Rees, M. J., 1978, In: Pittsbur gh Conference on BL Lac Objects, Pittsburgh, University of Pittsburgh, 1978, p. 328

Bloom, S. D., Marscher, A. P., 1996, ApJ, 461, 657

BŁażejowski, M. et al., 2000, ApJ, 545, 107

Burbidge, G. R. et al., 1974, ApJ, 193, 43

Catanese, M., Weekes, R. M., 1999, PASP, 111, 1193

Chiaberge, M., Ghisellini, G., 1999, MNRAS, 306, 551

Comastri, A. et al., 1997, ApJ, 480, 534

Crusius, A., Schlickeiser, R., 1986, A\&A, 164, L16

Dermer, C. D. et al., 1992, A\&A, 256, L27

Ghisellini, G. et al., 1988, ApJ, 334, L5

Ghisellini, G., Svensson, R., 1991, MNRAS, 252, 313

Ghisellini, G. et al., 1996, A\&AS, 120, 503

Giommi, P., Padovani, P., 1994, MNRAS, 268, L51

Hartman, R. C. et al., 1999, ApJS, 123, 79

Horan, D. et al., 2004, ApJ, 603, 51

Impey, C. D., Neugebauer, G., 1988, AJ, 95, 307

Kerrick, A. D. et al., 1995, ApJ, 452, 588

Koratkar, A. P. et al., 1998, ApJ, 492, 173

Lamer, G., Brunner, H., Staubert, R., 1996, A\&A, 311, 384

Maisack, M. et al., 1997, preprint, astro-ph/9706243

Maraschi, L. et al., 1992, ApJ, 397, L5

Massaro, E. et al., 1999, A\&A, 342, L49

Mattox, J. R. et al., 2001, ApJS, 135, 155

von Montigny, C. et al., 1995, ApJ, 440, 525

Rybicki, G., Lightman, A. P., 1979, Radiative Process in Astrophysics. Wiley Interscience, New York, P. 204

Sikora, M. et al., 1994, ApJ, 421, 153

Sreekumar, P. et al., 1996, ApJ, 464, 628

Stecker, F. W. et al., 2006, ApJ, 648, 774

Tagliaferri, G. et al., 2000, A\&A, 354, 431

Tavecchio, F., et al., 1998, ApJ, 509, 608 
Tosti, G. et al., 1999, ASPS, 159, 149

Ulrich, M. H. et al., 1997, ARA\&A, 35, 445

Urry, C. M., 1999, Astropart. Phys., 11, 159

Wolf, M., 1916, Astron Nachr., 202, 415

Worrall, D. M., Wilkes, B. J., 1990, ApJ, 360, 396 